\title{
SOME EXTENDED INTEGRAL INEQUALITIES ON TIME SCALES
}

\section{BOQUN OU}

Abstract. In this paper, we establish an integral inequality on time scales. As an applications, we study the stability of a kind of difference equations.

Mathematics subject classification (2010): 34N05,35B35.

Keywords and phrases: Integral inequality, time scales, stability.

\section{REFERENCES}

[1] L. Z. LI, F. W. Meng, P. J. Ju, Some new integral inequalities and their applications in studying the stability of nonlinear integro-differential equations with time delay, J. Math. Anal. Appl. 377 (2011) 853-862.

[2] M. Bohner, A. Peterson, Dynamic Equations on Time Scales: An Introduction with Applications, Birkhäuser, Boston, 2001.

[3] O. Lipovan, Integral inequalities for retarded Volterra equations, J. Math. Anal. Appl. 322 (2006) 349-358.

[4] B. G. Pachpatte, On a new inequality suggested by the study of certain epidemic models, J. Math. Anal. Appl. 195 (1995) 638-644.

[5] B. G. PACHPATte, Explicit bound on a retarded integral inequality, Math. Inequal. Appl. 7 (2004) 7-11.

[6] B. G. PachPatte, On a certain retarded integral inequality and its applications, J. Inequal. Pure Appl. Math. 5 (2004) (Article 19).

[7] M. Adivar, Elvan AKIn Bohner, Halanay type inequalities on time scales with applications, Nonlinear Analysis 74 (2011), 7519-7531

[8] L. Li, F. Meng, L. He, Some generalized integral inequalities and their applications, J. Math. Anal. Appl. 372 (2010) 339-349.

[9] N. T. J. BAILEY, The Mathematical Theory of Infectious Diseases and Its Applications, 2nd ed., Hafner, New York, 1975.

[10] QINGHUA MA, Estimates on some power nonlinear Volterra-Fredholm type discrete inequalities and their applications, J. Comput. Appl. Math. 233 (2010) 2170-2180.

[11] M. Bohner, Allan C. Peterson, editors, Advances in Dynamic Equations on Time Scales, Birkhäuser Boston Inc., Boston, MA, 2003.

[12] K. L. COOKE, I. GyORI, Numerical approximation of the solutions of delay differential equations on an infinite interval using piecewise constant arguments', Comp. Math. Appl. 28 (1994), 81-92.

[13] H. D. LiU, F. W. Meng, Nonlinear retarded integral inequalities on time scales and their applications, J. Math. Inequal. 12 (2018), 219-234.

[14] S. H. Saker, A. M. Ahmed, H. M. Rezk, D. O'Regan and R. P. Agarwal, New Hilbert dynamic inequalities on time scales, Math. Inequal. Appl. 20 (2017), 1017-1039.

[15] A. Tuna AND W. LiU, New weighted Cebysev-Ostrowski type integral inequalities on time scales, J. Math. Inequal. 10 (2016), 327-356.

[16] W. J. LiU, X. Y. GAO, Y. Q. WEN, Approximating the finite Hilbert transform via some companions of Ostrowski's inequalities, Bull. Malays. Math. Sci. Soc. 39 (2016), 1499-1513.

[17] Y. Jiang, H. RuZgar, W. LiU, A. Tuna, Some new generalizations of Ostrowski type inequalities on time scales involving combination of $\Delta$-integral means, J. Nonlinear Sci. Appl. 7 (2014), 311-324. 
[18] S. F. WANG, Q. L. XUE, W. J. LiU, Some new perturbed generalizations of Ostrowski-Grüss type inequalities for bounded differentiable mappings and applications, Appl. Math. Inf. Sci. 7 (2013), 2077-2081.

[19] Y. G. Sun, Some new integral inequalities on time scales, Math. Inequal. Appl. 15 (2012), 331-341. 